\title{
Prelinguistic Relational Concepts: Investigating Analogical Processing in Infants
}

\author{
Alissa L. Ferry, Susan J. Hespos, and Dedre Gentner \\ Northwestern University
}

\begin{abstract}
This research asks whether analogical processing ability is present in human infants, using the simplest and most basic relation-the same-different relation. Experiment $1(N=26)$ tested whether 7- and 9-montholds spontaneously detect and generalize these relations from a single example, as previous research has suggested. The attempted replication failed. Experiment 2 asked whether infants could abstract the relation via analogical processing (Experiment 2, $N=64$ ). Indeed, with four exemplars, 7 - and 9-month-olds could abstract the same-different relation and generalize it to novel pairs. Furthermore, prior experience with the objects disrupted learning. Facilitation from multiple exemplars and disruption by individual object salience are signatures of analogical learning. These results indicate that analogical ability is present by 7 months.
\end{abstract}

Analogical ability-the ability to make relational comparisons between objects, events, or ideas, and to think about relations independently of a particular set of arguments - is a cornerstone of higher reasoning abilities. Learning by analogy is a powerful and efficient method for acquiring new information. Equally important, analogical comparison facilitates the formation of abstract categories and rules (Gentner \& Medina, 1998; Gick \& Holyoak, 1983). Indeed, recent theoretical perspectives have asserted that analogical ability is the key capacity supporting higher order cognition and differentiating human cognitive capacity from that of other primates (Gentner, 2003, 2010; Penn, Holyoak, \& Povinelli, 2008).

Alissa L. Ferry is currently at Scuola Internazionale Superiore di Studi Avanzati.

This research was supported by NSF Grant BCS-1423917 to Susan J. Hespos, NSF SLC Grant SBE-0541957 awarded to the Spatial Intelligence and Learning Center (SILC), and by ONR Grant N00014-92-J-1098 to Dedre Gentner. Preparation of the article was supported in part by the European Research Council under the European Unions' Seventh Framework Programme (FP7/2007-2013)/European Research Council Grant Agreement 269502 (PASCAL), which supported Alissa Ferry during manuscript preparation and an award to Dedre Gentner from the Alexander von Humboldt Foundation and by a fellowship at the Hanse-Wissenschaftskolleg, Delmenhorst, Germany. We are indebted to parents who agreed to have their infants participate in the research. We thank members of the Infant Cognition Lab for help with data collection. Special thanks go to Don Tyrrell for his willingness to re-engage with these topics after 18 years of retirement. His thoughtful discussion has enhanced the quality of this work and has confirmed that these ideas retain their relevance across several decades. Finally, we thank Susan Carey for many insightful comments on this work.

Correspondence concerning this article should be addressed to Alissa L. Ferry, Cognitive Neuroscience Sector, Scuola Internazionale Superiore di Studi Avanzati, Via Bonomea 265, 34136 Trieste, Italy. Electronic mail may be sent to aferry@sissa.it.
Our goal in this research is to trace the development of relational processing - specifically, to discover whether processes of analogical learning are present in prelinguistic human infants. We focus on the same-different relation, for four reasons. First, it is arguably the simplest and most basic relation, and is likely to be available early in development. Second, the perception of sameness is critical to making sense of the world; in the words of William James (1890), the "sense of sameness is the very keel and backbone of our thinking" (p. 459; quoted in Wasserman \& Young, 2010). A third point is that the same-different relation has the advantage that it can be tested by a simple perceptual task: the twoitem same-different task, which is readily adaptable for infant studies. If a participant can discriminate between same pairs $\{\mathrm{AA}, \mathrm{BB}, \mathrm{CC} .$.$\} and different$ pairs $\{A B, C D, E F .$.$\} and can generalize this dis-$ tinction to new exemplars ( $X X$ vs. $Y Z$ ), then we can infer that they have represented abstract same and different relations.

The final reason for focusing on the same-different relation is that it has been the main focus of comparative research on relational ability in nonhuman species. Beginning with Premack's (1983) seminal paper, which argued that chimpanzees (but not monkeys) possess significant relational ability, much of this research has focused on the same-different relation (Fagot \& Thompson, 2011; Flemming,

(C) 2015 The Authors

Child Development (c) 2015 Society for Research in Child Development, Inc. All rights reserved. 0009-3920/2015/8605-0006

DOI: $10.1111 /$ cdev.12381 
Beran, \& Washburn, 2007; Giurfa, Zhang, Jennett, Menzel, \& Srinivasan, 2001; Thompson, Oden, \& Boysen, 1997; Wasserman \& Young, 2010). This research has shown that although many species, including pigeons, macaques, and honeybees, can pass the object match-to-sample test (given a standard A, choose the matching alternative, A, over $\mathrm{B})$, the ability to pass the same-different task (making one response for $\mathrm{AA}, \mathrm{BB}, \mathrm{CC}$, etc. and another for $A B, C D, E F$, etc.) is sharply graded across species. For example, Wright and Katz (2006) found that rhesus monkeys and capuchin monkeys required over 4,700 trials to learn to distinguish same pairs from different pairs and generalize the distinction to novel pairs. These findings show the difficulty of same-different relational matching for monkeys. In contrast, chimpanzees can learn and generalize the same-different relation in 150-500 trials (Premack, 1983; Robinson, 1955) and human 3-year-olds given a sorting task can learn to distinguish same and different relations after fewer than 24 trials (Christie \& Gentner, 2014). A more challenging task, in which the subject must match a same pair to either a same (correct) or a different pair, was given to baboons (Fagot \& Thompson, 2011). Only 6 of 29 animals were able to reach criterion, and they required a mean of 21,000 trials. In contrast, human 4-year-olds succeed at this task (for the same relation) in just eight trials without feedback (Christie \& Gentner, 2014). Thus, relational ability - even with the basic same-different relationvaries strongly across primate species.

\section{The Ontogeny of Human Analogical Ability}

Adult humans - and even children-are virtuosos of relational ability compared with other primates. But how does this ability develop?

How do infants come to be able to encode and generalize relations between things? We can distinguish three broad positions. The strongest possibility is that human infants are born with a set of privileged relations that they naturally use to encode experience-a set of relational semantic primitives. Such a set would almost certainly include the samedifferent relation. In this case, infants would be able to encode abstract same and different relations even from a single exemplar. A second possibility is that human infants are born with analogical ability, with which they can learn abstract relations from experience, but without a starting set of abstract relations. In this case, infants would be unable to spontaneously encode the same and different relations from a single exemplar but might be able to use analogical learning mechanisms to encode these relations by comparing exemplars. A third possibility is that analogical ability is not inherent in human biology, but develops by combining other abilities through cultural and linguistic experience. This possibility receives some support from studies that show that learning and using relational language facilitates relational insight in children (Christie \& Gentner, 2014; Gentner, 2003, 2010; Kotovsky \& Gentner, 1996). In this case, we would not expect to see evidence of abstract same and different relations until children have acquired a degree of cultural and linguistic experience- perhaps including some knowledge of relational terms.

The first and strongest possibility is that same and different are part of an innate "starter kit" of abstract relational primitives, akin to the semantic features postulated by some early theories of meaning acquisition (e.g., Clark, 1973). This account also assumes that infants are born with analogical ability - that is, the ability to align and map between relations. We consider this possibility in Experiment 1 , and then turn to the other two possibilities.

The idea that abstract same and different relations are present from the start has had wide influence, particularly in comparative psychology, through the pioneering work of Tyrrell, Stauffer, and Snowman (1991). This research suggested that 7-month-old humans spontaneously encode and transfer same and different relations from a single exemplarimplying that human infants can encode abstract same-different relation without training. Because of the importance of these claims, our first order of business was to examine and replicate this study. Unfortunately (to preview) our attempted replication failed; further, inspection of the paper revealed that it does not support the strong possibility above.

We first review this study and then present our attempted replication. Tyrrell et al. (1991) tested twenty-two 7-month-old infants. Half of the infants were familiarized to an exemplar of the same relation (AA; presented twice); the other half were familiarized to an exemplar of the different relation $(\mathrm{BC})$, also presented twice. In the first test trial, infants were shown a side-by-side presentation of $\mathrm{AA}$ and $\mathrm{BC}$ - that is, the pair they had seen along with a pair they had not seen (and which depicted a different relation from the familiar pair)-until $10 \mathrm{~s}$ of looking at either pair accumulated. In the second test trial, they saw two new pairs (DD and $\mathrm{EF})$, and the relative positions of the same and different pairs were switched. The reasoning was if the infants showed a novelty preference for the relation that they had not been familiarized to, this would 
indicate that they already possess an abstract same-different relation, which can be generalized from a single exemplar. Combining both of these test trials, Tyrrell et al. (1991) found just this pattern: After habituation to same the infants looked significantly longer at the novel relation (different) in the test trials than at the familiar relation (same). The same novelty preference was also found for infants habituated to different. Because the infants had seen only one instance of the relation prior to testing, this seemed to suggest that 7-month-olds spontaneously encode abstract relational conceptspotentially a very important result. Given the importance of this question, and the oft-cited interpretation that their study represents evidence of generalizing same-different relations from a single exemplar, we set out to replicate the experiment using a single exemplar, to test whether infants can spontaneously detect the same-different relations.

Before turning to the replication, we note that the original Tyrrell et al. (1991) publication contains some ambiguities regarding the exact method. However, subsequent discussions of this influential work have adopted the interpretation offered here -namely, that infants generalized same (or different) from a single example - indicating that they already possessed abstract representations of same and different (e.g., Dewar \& Xu, 2010; Marcus, Fernandes, \& Johnson, 2007; Thompson \& Oden, 1995, 2000; Tyrrell, Zingardo, \& Minard, 1993; Zentall, Wasserman, Lazareva, Thompson, \& Rattermann, 2008).

\section{Experiment 1}

In this study, we set out to replicate the research of Tyrrell et al. (1991). Our goal was to discover whether 7-month-old infants would encode an abstract same (or different) relation from a single familiarization exemplar. We also tested a group of 9-month-old infants to capture potential developmental differences, as previous research had suggested that by this age, infants show some evidence of understanding same and different in a different task (Addyman \& Mareschal, 2010). We followed an interpretation of Tyrrell et al.'s experimental design with only a single familiarization exemplar. As shown in Figure 1A, during familiarization, infants saw two presentations of the same example pair (e.g., AA). In the first test trial (old), they chose between this initial example pair (AA) and a new pair depicting the other relation (e.g., BC). In the second test trial (novel), same and different were instantiated with new objects. Only if infants differentiate the relations on the second trial can we infer that they already possess abstract same-different relations.

\section{Method}

\section{Participants}

The participants were 26 healthy, full-term infants, ten 7 -month-olds (5 male and 5 female) ranging from 6 months 23 days to 7 months

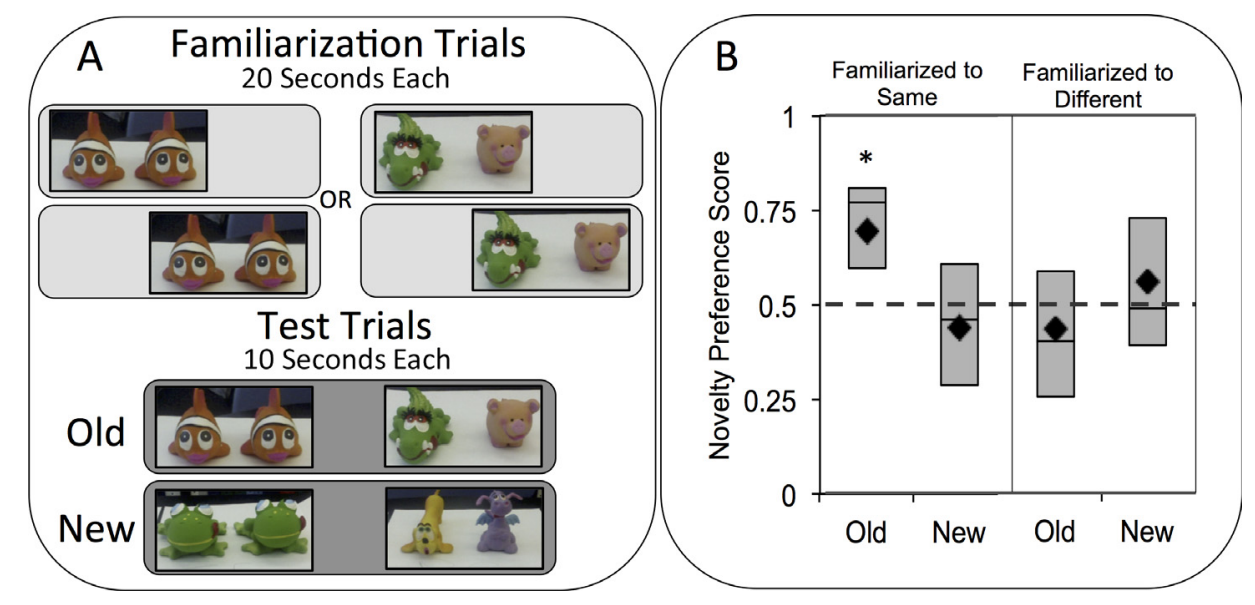

Figure 1. (A) Schematic of Experiment 1. During familiarization trials, infants were shown either a pair of same or a pair of different objects. During the test trials, infant saw two pairs of objects presented side by side. For the old test trial, one pair was identical to the pair seen during familiarization; the other pair consisted of novel objects and a novel relation. For the new test trial, all the objects were novel and one pair depicted the familiarized relation and the other depicted the novel relation. (B) Test trial novelty preference scores in Experiment 1. Box plots showing mean preference score for the novel relation over the familiar relation during both test trials. Black diamonds represent means, the central line in each box is the median, and the upper and lower portions of each box represent the third and first quartiles, respectively. The dashed line represents chance performance.

${ }^{*} p<.05$. 
24 days $(M=7$ months 4 days $)$ and sixteen 9month-olds (6 male and 10 female) ranging from 8 months 19 days to 9 months 16 days $(M=$ 8 months 29 days). Approximately half the infants were assigned to the same condition $(n=11)$; the other half, to the different condition $(n=15)$. Four additional infants were tested but eliminated from the final analysis, three because of parental interference and one because he looked only at the right side of the stage during every test trial.

For this and the following experiment, we obtained infants' names from commercial mailing lists. Parents were contacted by letters and provided informed consent before the experiment. They were given $\$ 20$ as compensation. The ethnicity of the sample was $81 \%$ non-Hispanic, $14 \%$ Hispanic, and the remaining $5 \%$ chose not to answer. The racial makeup was $76 \%$ white, $6 \%$ Black/African American, 4\% Asian, 1\% Native Hawaiian/ Pacific Islander, 1\% American Indian/Alaskan Native, $7 \%$ multiracial. The remaining $5 \%$ chose not to answer. The mothers' highest level of education included 1\% some high school, $6 \%$ high school diploma, $14 \%$ some college, and $75 \%$ college degree or higher; the remaining $4 \%$ chose not to answer. The fathers' highest level of education included 1\% some high school, $4 \%$ high school diploma, $11 \%$ some college, $80 \%$ college degree or higher; the remaining $4 \%$ chose not to answer.

\section{Apparatus}

Parents sat in a chair with infants on their lap facing a wooden puppet stage that displayed all stimulus objects. The stage measured $243.5 \mathrm{~cm}$ high, $128 \mathrm{~cm}$ wide, and $61 \mathrm{~cm}$ deep. The opening in the front of the stage that displayed the objects was $93 \mathrm{~cm}$ above the floor, $61 \mathrm{~cm}$ high, and $106 \mathrm{~cm}$ wide. The back wall had two rectangular openings with cloth fringe over the openings that allowed the experimenter to manipulate the objects between trials. A screen that covered the infants' view of the stage was raised and lowered between trials (see Figure 1A).

The stimuli consisted of four pairs of objects, each attached by Velcro to a $26.5 \mathrm{~cm} \times 15.5 \mathrm{~cm}$ piece of cardboard covered with contact paper. The items in each pair were approximately $12.5 \mathrm{~cm}$ apart from each other. Two pairs of objects instantiated the same relation (two identical fish and two identical frogs) and two pairs of objects instantiated the different relation (a crocodile and a pig paired together and a dog and a dragon paired together).

\section{Events}

Familiarization trials. When the screen went up at the start of a trial, there was a pair of objects on either the right or left side of the stage. Half of the infants were shown the objects with the same relation while the other half of the infants were shown the objects with the different relation. The objects remained stationary on the stage until the infant accumulated $20 \mathrm{~s}$ of looking time at the pair, after which the screen was lowered. The pair was moved to the opposite side of the stage and the familiarization trial was repeated. Across the familiarization trials, infants accumulated $40 \mathrm{~s}$ of total looking at the pair.

Test trials. Two types of test trials were presented in sequence to all participants. The first test trial (old) consisted of the pairs from familiarization (fish-fish and crocodile-pig) placed on opposite sides of the stage for $10 \mathrm{~s}$ of accumulated infant looking. As in Tyrrell et al. (1991) study, the infants had seen one of these pairs before. The second test trial (novel) consisted of new object pairs (e.g., frog-frog as the same relation and dog-dragon as the different relation) placed on opposite sides of the stage for $10 \mathrm{~s}$ of accumulated infant looking. The amount of time infants fixated on each object pair for each test trial was recorded.

\section{Procedure}

During the experiment, the infant sat on the parent's lap in front of the apparatus. The parents were asked to refrain from interacting with their infant during the experiment and to close their eyes during the test trials. All trials ended when the infant accumulated the necessary amount of looking time during each trial $(20 \mathrm{~s}$ total infant looking for each familiarization trial and $10 \mathrm{~s}$ for each test trial). A computer determined the end of the trial and signaled the experimenter to lower the screen. The initial placement (left or right side of the stage) of the familiarization and test stimuli was counterbalanced across infants.

\section{Coding}

There was a small hole in the front face of the stage containing a camera that captured a video image of the infant's face. Two research assistants in a separate room viewed this image and coded infants' visual fixations online. Each researcher depressed a computer button when the infant attended to the objects on stage and released the button when the infant looked away. Later, offline 
frame-by-frame coding to determine left and right looks was conducted using ELAN (2007). Thirtythree percent of infants in each age and condition were recoded by an independent observer. Reliability between observers averaged $92 \%$ during familiarization and 91\% during test. Three infants (two familiarized to same and one familiarized to different) looked exclusively to one test stimulus on one of the test trials. Because we were uncertain whether these infants noticed the other test stimulus, infants were given a 2-min break to play in the waiting room and the familiarization and test trials were rerun with a completely different set of objects. We obtained the same results (see below) whether we included these preference scores for these infants or included the original, exclusive test trials.

To measure categorization, a preference score was calculated for each infant by dividing their looking time at the novel-category exemplar during test by their total looking time (10 s).

\section{Results}

Preliminary analyses revealed no significant effect of age or sex on the infants' looking times, nor any interactions involving these variables. The subsequent analyses therefore collapsed across these variables. The results, depicted in Figure 1B, reveal that infants familiarized to same showed a novelty preference in the old test trial, but no preference in the novel test trial. Infants familiarized to different did not differ from chance in either test trial. Overall, infants were not able to abstract the same-different relation to the novel pairs, and only those infants familiarized to same showed a novelty preference even on the old test trials.

These patterns are borne out by an analysis of variance (ANOVA) on infants' preference scores at test, with condition as a between-subject factor (familiarized to same or different) and test trial (old or novel) as a within-subject factor. This revealed no main effects, but it did reveal a significant Condition $\times$ Test Trial interaction, $F(1,24)=5.62, p=.026, \eta^{2}=.19$. Further analyses revealed that within the test trials, infants' performance varied as a function of their familiarization condition, $F(1,24)=8.60, p=.007$. In the old test trial, infants familiarized to same showed a significant novelty preference $(M=0.70, S D=0.18)$, $t(10)=3.577, p=.005$, while infants familiarized to different did not significantly differ from chance $(M=0.43, S D=0.25), \quad t(14)=-1.007, p=.33$. In contrast, within the novel test trials, performance did not differ between the two familiarization groups,
$F(1,24)=1.53, p=.23$; neither the infants familiarized to same $(M=0.45, S D=0.20), t(10)=-0.84$, $p=.42$, nor the infants familiarized to different $(M=0.56, S D=0.24), t(14)=0.96, p=.35$, were significantly different from chance.

\section{Discussion}

Our attempted replication of the Tyrrell et al. (1991) experiment failed to find evidence that infants encode abstract same-different relations from a single training exemplar. That is, there was no generalization to novel test pairs, either for infants familiarized to the same pair or for those familiarized to the different pair. The only significant finding was that infants familiarized with the same pair looked significantly longer at the different pair-but only during old test trials, when the same pair was identical to the pair seen in familiarization. Infants familiarized to the different pair showed chance performance in both test trial types. Thus, we have no grounds to conclude that 7- or 9-month-olds spontaneously encode abstract same (or different) relations.

The findings by Tyrrell et al. (1991) have been taken as evidence that humans naturally encode abstract relations from very early in development, and may be endowed with a primitive set of abstract relations. Our nonreplication, together with the fact that Tyrell et al.'s studies were inconclusive on this point, calls for a reconsideration of this question. Our findings fail to show support for the hypothesis that infants are endowed with same-different relations. At the very least, it appears that these relations are not immediately available to the infant.

Earlier we laid out three possibilities for how analogical ability might develop. The first two both assume that analogical ability is an innate capacity of our species. Possibility 1 adds the assumption that infants are born with a set of primitive relations, including the same-different relation. This possibility can be set aside, at least as a solution for how same and different are acquired. We now turn to the other two possibilities. Possibility 2 is that infants begin life with analogical learning processes that enable them to abstract relations from experience. Possibility 3 is that analogical ability is not an inherent capacity but is formed by combining more basic processes, guided by cultural and linguistic experience.

We now turn to the second possibility: that infants begin life with analogical learning processes that enable them to abstract relations from experience. 
Experiment 2 will test this possibility. If we find that 7-month-olds can abstract same and different relations through analogical abstraction processes, then Possibility 2 will remain a viable explanation. If not, then we will be left with Possibility 3 .

Although little is known about the early origins of human analogical ability, there has been considerable research on the development of analogical ability from preschool to adulthood. The available evidence suggests that the same basic analogical mapping processes occur for young children as for older children and adults (Gentner \& Medina, 1988, 2003, 2010; Gentner \& Namy, 1999; Mix, 2008; Richland, Morrison, \& Holyoak, 2006).

Another parallel between children and adults is that the presence of salient objects can interfere with relational mapping. This propensity is especially pronounced early in development. Young children often focus on object matches, whereas older (or more knowledgeable) children focus on relational matches (Gentner \& Toupin, 1986; Kotovsky \& Gentner, 1996; Paik \& Mix, 2006; Richland et al., 2006; Smith, 1984) - a pattern that has been described as the relational shift (Gentner, 1988; Gentner \& Rattermann, 1991). This shift has been attributed to increases in relational knowledge (Gentner, 2003; Gentner \& Rattermann, 1991), to maturational increases in processing capacity (Halford, 1992), and to increases in executive ability, including inhibitory control (Doumas, Hummel, \& Sandhofer, 2008; Richland et al., 2006; Thibaut, French, \& Vezneva, 2010), and it is possible that all three play a role.

A signature component of analogical learning is that the ability to perceive abstract relational matches can be enhanced by comparing different instances of a relation. For example, Gick and Holyoak (1983) found that comparing two stories that shared causal structure enabled people to generalize that structure and to transfer it to a further situation. These findings are consistent with other research suggesting that the act of comparison entails a structural alignment process that highlights the relational commonalities between the items compared (Gentner \& Medina, 1998; Markman \& Gentner, 1993). Similar effects of comparison have been found for preschool children (Gentner, Anggoro, \& Klibanoff, 2011; Gentner \& Namy, 1999; Kotovsky \& Gentner, 1996).

The influence of structural alignment in promoting relational focus is a defining characteristic of analogical reasoning in adults (Gentner, Holyoak, \& Kokinov, 2001), and the evidence of its influence in children as young as 3 years of age suggests that there may be continuity in analogical learning processes through human development. A second signature component of analogical learning is that attention to individual objects can interfere with relational processing. This is especially true for young children, who show a strong tendency to focus on objects rather than relations. Preschool children perform far worse on relational matching tasks when competing object matches are present (Gentner \& Toupin, 1986; Richland et al., 2006), especially if the objects involved are rich and distinctive (DeLoache, 1995; Gentner \& Rattermann, 1991; Paik \& Mix, 2006). The finding that attention to objects can overshadow attention to relations extends to very young age groups (Casasola, 2005; Maguire, Hirsh-Pasek, Golinkoff, \& Brandone, 2008).

A study by Christie and Gentner (2010) illustrates both the appeal of object matches to young children and the effect of comparison in promoting attention to common relational structure. Christie and Gentner taught 3-year-olds names for novel spatial relational patterns, and asked them to choose a further instance of the pattern (see Figure 2). Children given only one standard extended the terms on the basis of object matches rather than common relational structure. For example, given a
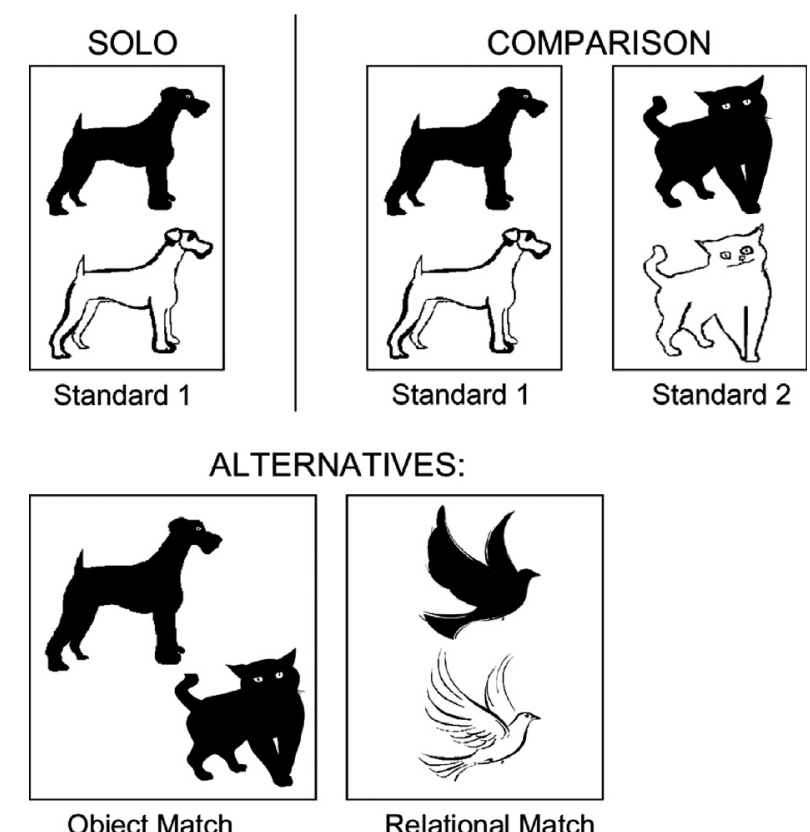

Figure 2. Sample set used in Christie and Gentner (2010) study. Children who saw one standard (either Standard 1 or Standard 2) overwhelmingly chose the object match those who compared the two standards were more likely to choose the relational match. 
standard black dog above white dog; otherwise identical, the children would choose an alternative showing a black dog and a black cat, rather than one showing the same relational pattern instantiated with different objects. Across two studies, over $90 \%$ of the 3-year-olds' responses were to the object matchdemonstrating the strength of the object bias in young children. However, when a new group of 3year-olds was led to compare two standards showing the same relational pattern, the majority of their responses were to the relational match. The effects of comparison were quite striking: The proportion of relational matches rose from $2 \%$ to $57 \%$-even though the competing object alternative contained two object matches.

\section{Prior Evidence on Early Relational Insight}

There is considerable evidence that infants possess some understanding of spatial relations (Casasola, 2005; Casasola \& Cohen, 2002; Hespos, Grossman, \& Saylor, 2010; Hespos \& Piccin, 2009; Hespos \& Spelke, 2004; McDonough, Choi, \& Mandler, 2003; Quinn, Cummins, Kase, Martin, \& Weissman, 1996) and physical relations (Hespos \& Baillargeon, 2001, 2006; Wang \& Baillargeon, 2008; for a review, see Baillargeon, Li, Gertner, \& Wu, 2011). Much of this work has focused on infants' knowledge about interactions between objects. In many of these studies - particularly those using the violation-of-expectation paradigm - the goal is to trace the early development of insight into spatial and physical processes. Although some of this work has investigated the processes involved in gaining knowledge (e.g., Casasola, 2005; Quinn et al., 1996; Wang \& Baillargeon, 2008), in most cases the focus has been on discovering what knowledge infants bring to the laboratory. In contrast, our goal is to study infants' learning processes during the experiment itself. We ask whether infants can derive abstract same-different relations from specific exemplars by processes of analogical abstraction.

Perhaps the clearest case of relational learning in infants comes from artificial grammar tasks (Aslin \& Newport, 2012; Saffran, Aslin, \& Newport, 1996), particularly those in which infants learn a relational pattern that can be transferred to new exemplars (Gerken, 2006; Gomez \& Gerken, 2000; Johnson et al., 2009; Kirkham, Slemmer, \& Johnson, 2002; Marcus, Vijayan, Rao, \& Vishton, 1999). For example, in the Marcus et al. (1999) study, when 7.5month-olds heard 48 examples (16 patterns, three times each) of a syllable pattern such as $\mathrm{AAB}$, they could then discriminate new instances of the $\mathrm{AAB}$ pattern from instances of an ABA pattern, even when all the specific syllables were new (see also Gomez \& Gerken, 1999). Furthermore, there is evidence that the ability to generalize across such patterns is not restricted to language-like materials; it can operate across a broad range of stimuli, including tones and visual stimuli (Gomez \& Gerken, 2000; Johnson et al., 2009; Saffran, Pollak, Seibel, \& Shkolnik, 2007).

These experiments focus on learning a particular pattern that emerges during the course of the experiments. How might this be done? Marcus (2000) notes two main approaches-one based on assuming that the child is trying to predict the next syllable, and the other based on assuming a preprocessor that transforms each input item into an abstract form. We suggest a third possibilitynamely, that analogical learning mechanisms might take concrete descriptions as input and generalize across a sequence of items to arrive at a more abstract representation. In support of this possibility, Kuehne, Gentner, and Forbus (2000) showed that SEQL (sequential learning engine), a computational model of analogical generalization, can capture the Marcus et al. (1999) findings. SEQL is an offshoot of SME (structure-mapping engine), and operates by using SME to iteratively compare input examples to an ongoing generalization. The model was given the same input as the infants in Marcus et al.: three repetitions of each of the 16 strings, with each syllable encoded as 12 phonemic features (following Elman, 1998). The relational pattern within each string (e.g., AAB) was encoded by Magi, which uses SME to encode symmetry and repetition within an item (Ferguson, 1994). As the strings were presented, SEQL computed a generalization by comparing the first two exemplars (via SME) and storing their common structure, and then incrementally comparing subsequent exemplars to the ongoing abstraction. After three (randomly ordered) repetitions of each example string, SEQL was given two test strings with new syllables. Like the infants in Marcus et al., it was found that the test string with the same relational structure (e.g., CCD) was more similar to its generalization than the one with different structure (e.g., CDC). This rule-like behavior emerged despite the fact that no variables were introduced, and in every run of the simulation, some concrete features were still present at the end. These results provide evidence that analogical generalization could capture the emergence of grammatical patterns. However, because the artifi- 
cial grammar studies were not designed to test for analogical processes, evidence for (or against) specific signatures of analogical processing is lacking. We return to these issues in the General Discussion.

\section{The Same-Different Relation in Infants}

Few studies have looked directly at whether infants can detect same-different relations. We know from the artificial grammar studies just discussed that infants can discriminate $\mathrm{AAB}$ patterns from ABA patterns (Marcus et al., 1999; Saffran et al., 2007), but this does not tell us whether the infants could distinguish AA from AB. That is, the ability to recognize a pattern does not imply the ability to recognize all of its individual subpatterns (Palmer, 1977; Sinha, Balas, Ostrovsky, \& Russell, 2006).

More recently, Addyman and Mareschal (2010) showed infants of 4 and 8 months nonrepeating exemplars of either same or different (up to a maximum of 19 exemplars) during familiarization. Then the infants viewed test trials, presented sequentially. They reported that 8 - but not 4-month-olds infants showed evidence of looking longer at the novel than at the familiar relation. It is difficult to interpret these results, because half the infants in both age groups had participated in a prior samedifferent study during the same session. However, it is interesting that although both age groups received over 18 familiarization trials, very few infants showed habituation (a decline in looking time over the course of trials), suggesting that the same-different relation did not emerge readily. Still, these results provide encouragement for the idea that by 8 months of age, infants can distinguish same and different relations under certain conditions. We now turn to the question of how infants extract relational commonalities.

\section{Experiment 2}

In Experiment 2, we tested the possibility that infants' relational learning stems from analogical processing - that is, carrying out structural alignment across exemplars and thereby extracting common relational structure. The question then becomes: Can infants derive abstract same-different relations from a brief series of exemplars, and if so does this learning process bear the signatures of analogical learning? That is, we ask whether infants engage analogical learning mechanisms during the learning phase to extract relational commonalities from a series of exemplars.

As reviewed above, two key characteristics of analogical learning are: (a) that comparing relationally similar exemplars promotes relational abstraction and (b) that focusing on individual objects interferes with relational processing. In Experiment 2, we ask whether infants would show this learning pattern. That is, we ask, first, whether 7- and 9-month-olds could successfully abstract same or different relations from multiple exemplars, generalizing the relation to exemplars composed of completely new objects. Such a pattern would suggest that the ability to align sequential exemplars and extract their common relation-a signature of analogical processing in older children-is evident in infants as well. Second, we ask whether infants would also show adverse effects of object focus. If object focus distracts from relational processing, this would constitute a further parallel with analogical learning in older children.

In Experiment 2, infants received training on either same or different relations. During testing, infants saw pairs instantiating the same and different relation. The question was whether they would differentiate the familiar relation from the new relation. Critically, each infant saw three kinds of test pairs (as amplified below). Some test pairs were made up of entirely new objects; these tested whether infants had abstracted the relation across the habituation pairs. A second kind of test pairs consisted of objects that had been rendered salient earlier in the session; these tested whether object salience would disrupt relational processing, as in older populations. The third kind of test pairs consisted of objects that had been rendered salient but that had subsequently appeared in the habituation series. These pairs tested whether repeated alignment across pairs would overcome initial object salience, as has also been found for older children. Finally, our method also allowed us to ask whether infants are equally proficient at learning same and different relations.

The key predictions from the analogical learning account are (a) that alignment across relationally identical pairs should render the common relation more salient and promote abstracting the relation and (b) that attention to objects should disrupt structural alignment and therefore interfere with relational abstraction. Thus, if infants are learning via analogical processes, they should differentiate the familiar relation (e.g., same, if habituated to same) from the unfamiliar one (e.g., different) for novel test pairs. 


\section{Method}

\section{Participants}

The participants were 64 healthy, full-term infants, thirty-two 7-month-olds (17 male and 15 female) ranging from 6 months 9 days to 7 months 22 days $(M=6$ months 28 days) and thirty-two 9-month-olds (13 male and 18 female) ranging from 8 months 15 days to 9 months 19 days $(M=$ 9 months 4 days). Half the infants were assigned to the same condition; the other half, to the different condition. Seven additional infants were tested but eliminated from the final analysis - three because of fussiness, three because of parental interference, and one due to inattentiveness. The recruitment and socioeconomic status information was similar to Experiment 1.

\section{Apparatus}

The stage setup was identical to that used in Experiment 1. However, for this experiment we adopted a habituation-dishabituation paradigm to allow us to examine the rate of learning during training trials. Another change was that the same and different test trials were presented sequentially, rather than side by side. XHab (Pinto, 1996) was used to record looking times for habituation and test trials during the experiment.

The stimuli consisted of 16 objects, composed of 10 distinctive types (Elmo, blue fuzzy head, pink dotted block, checkered cylinder, dragon, red striped block, camel, pig, green puff balls on wood, and red/green foam block), with each pair placed on a $26.5 \times 15.5 \mathrm{~cm}$ piece of cardboard covered with contact paper (see Figure 3). Twelve of the objects (composed of seven distinct types) were presented in the playroom before the experiment began. Of these, eight (four pairs) were also used in the habituation phase and in the test phase, and three were used in the test phase only. Four pairs of objects were used in habituation and six were used in the test.

\section{Events}

Object experience phase. In the object experience phase, we manipulated object focus by showing some of the test objects to the infants in the playroom prior to the experiments, during naturalistic play interactions. We held up the objects and made comments such as, "Look!" and "See this one?" The objects were presented individually and in no

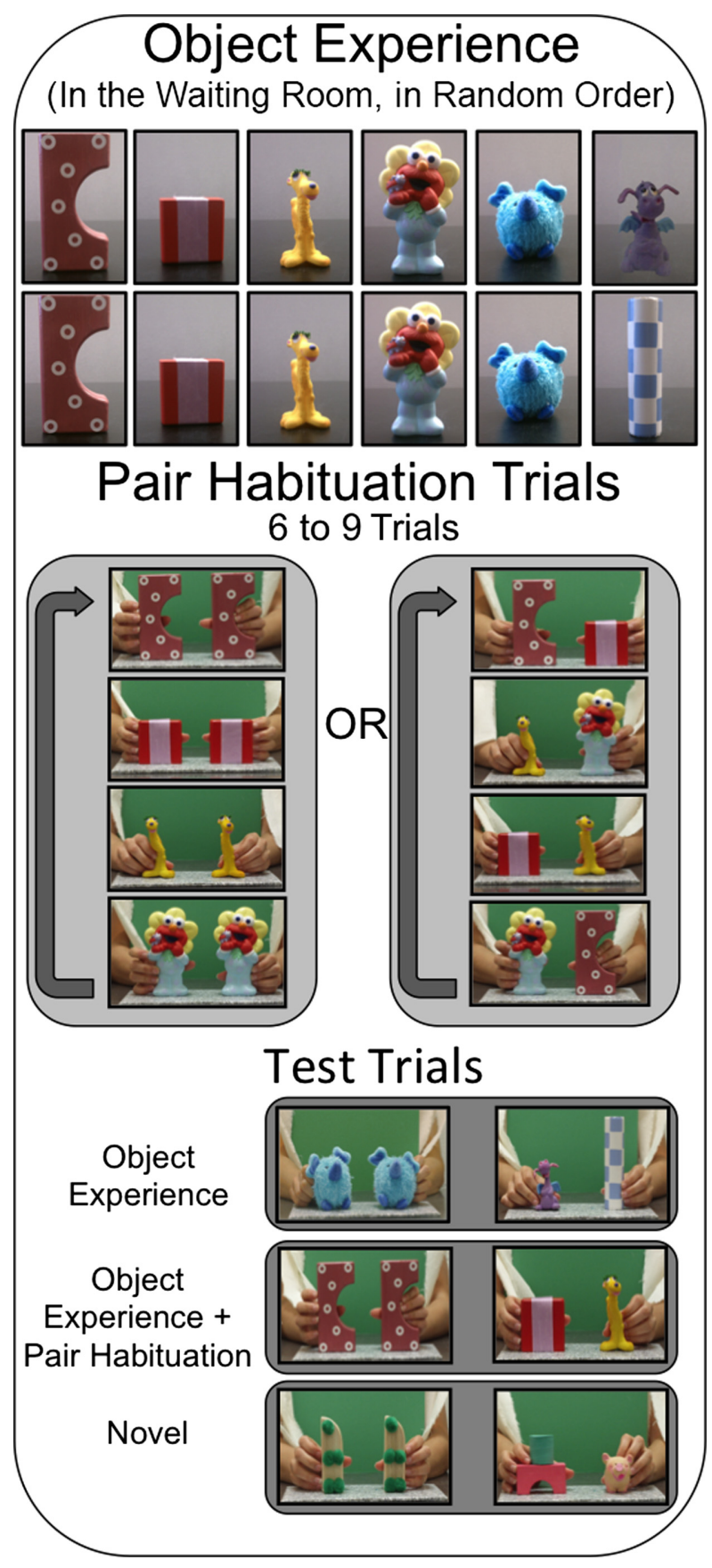

Figure 3. Schematic of events in Experiment 2. In the waiting room infants saw a subset of the individual toys before the experiment. Infants were habituated to four pairs of objects, either same or different. In six sequential test trials looking time was recorded to the novel and familiar relational pairs in three different types of test trials (object experience, object experience + pair habituation, and novel).

particular order for approximately $5 \mathrm{~s}$ for each object. The objects consisted of all of the objects used in the habituation trials (two of each Elmos, 
camels, red blocks, and pink blocks), as well as a set of objects used only in the object experience test trials (two blue fuzzy heads, one checkered cylinder, and one dragon).

Pair habituation trials. To engage infants' attention, in both habituation and test trials the pairs were moved during the trial. When the screen went up at the start of a trial, a pair of objects rested on the cardboard plate on the stage. Each of the experimenter's hands grasped one of the objects in the pair and raised the objects straight up ( $1 \mathrm{~s})$, tilted the objects to the left $(1 \mathrm{~s})$, returned them to the center $(1 \mathrm{~s})$, tilted them to right $(1 \mathrm{~s})$, returned them to the center (1 s), returned them to the plate (1 s), and paused on the plate $(2 \mathrm{~s})$. This 8 -s cycle repeated continuously until the trial ended. In the same condition, infants saw habituation trials in which the pairs of objects were the same (see Figure 3). In the different condition, infants saw habituation trials in which the pairs of objects were different. Infants saw four sets of object pairs during habituation trials. These four pairs were repeated until the infant reached the habituation criterion. The order of presentation was counterbalanced across infants.

Test trials. Infants received six test trials. In each test trial, infants viewed one pair of objects, presented in the same manner as in the habituation trials, while their looking time was recorded. Each infant received test trials with both same and different pairs of objects, presented in alternation, with order counterbalanced across infants. There were three kinds of test trials: (A) pairs consisting of objects that the infant had only experienced individually in the playroom (object experience), (B) pairs consisting of objects that had been seen individually in the playroom and then in pairs during habituation (object experience + pair habituation), and (C) pairs consisting of objects that were completely novel, in that the infants saw them for the first time during the test trials (novel). There were three trial orders in which the test items could appear: $A B C$, $\mathrm{BCA}$, or $\mathrm{CAB}$. This allowed each type of test pair to be first for a third of the infants. This ensured that the order of the test pairs was counterbalanced across infants and that any effects of test trial type were distinguishable from effects due to the order of the test pairs.

\section{Procedure}

During the experiment, the infant sat on the parent's lap in front of the apparatus. The parents were asked to refrain from interacting with their infant during the experiment and to close their eyes during the test trials. All trials ended when the infant either looked away for 2 consecutive seconds after having looked at the event for at least $2 \mathrm{~s}$, or looked at the event for 60 cumulative seconds without looking away for 2 consecutive seconds. A computer determined the end of the trial and signaled the experimenter to lower the screen. The habituation criterion was at least a $50 \%$ decline in total looking duration from the first three to the last three habituation trials or a maximum of nine trials. Each infant viewed six test trials, alternating between same or different pairs of objects. The type of test event shown first and the order of the test trials was counterbalanced across infants. Interobserver agreement was measured for all infants and averaged $94 \%$ per trial per infant.

\section{Results}

Preliminary analyses revealed no significant effect of gender or test trial order on the infants' looking times; the subsequent analyses therefore collapsed across these variables.

\section{Overall}

The first analyses addressed our central question: whether infants are capable of abstracting same and different relations from multiple exemplars. The answer appears to be yes; infants looked significantly longer at the novel relation than at the familiar relation during the test trials for both same and different (see Figure 4). Collapsing across all test trial types, 49 of the 64 infants $(77 \%)$ had longer looking times for the novel test events than for the familiar test events ( $p<.001$, binomial comparison). Across all participants, the average looking times were $18.32 \mathrm{~s}(S D=8.34)$ for the novel relations and $14.50 \mathrm{~s}(S D=7.05)$ for the familiar relations, $F(1$, $63)=19.87, p<.001, \eta^{2}=.24$. Overall, infants detected a change from the familiar to the novel relation.

\section{Effects of Prior Experience}

The next set of analyses tested whether prior experience in which attention was called to individual objects would impede relational learning for pairs containing these objects; and if so, whether this adverse effect would be mitigated by exposure to pairs containing these objects during habituation. In both cases, the answer appears to be yes. An ANOVA comparing the within-subject factors of 

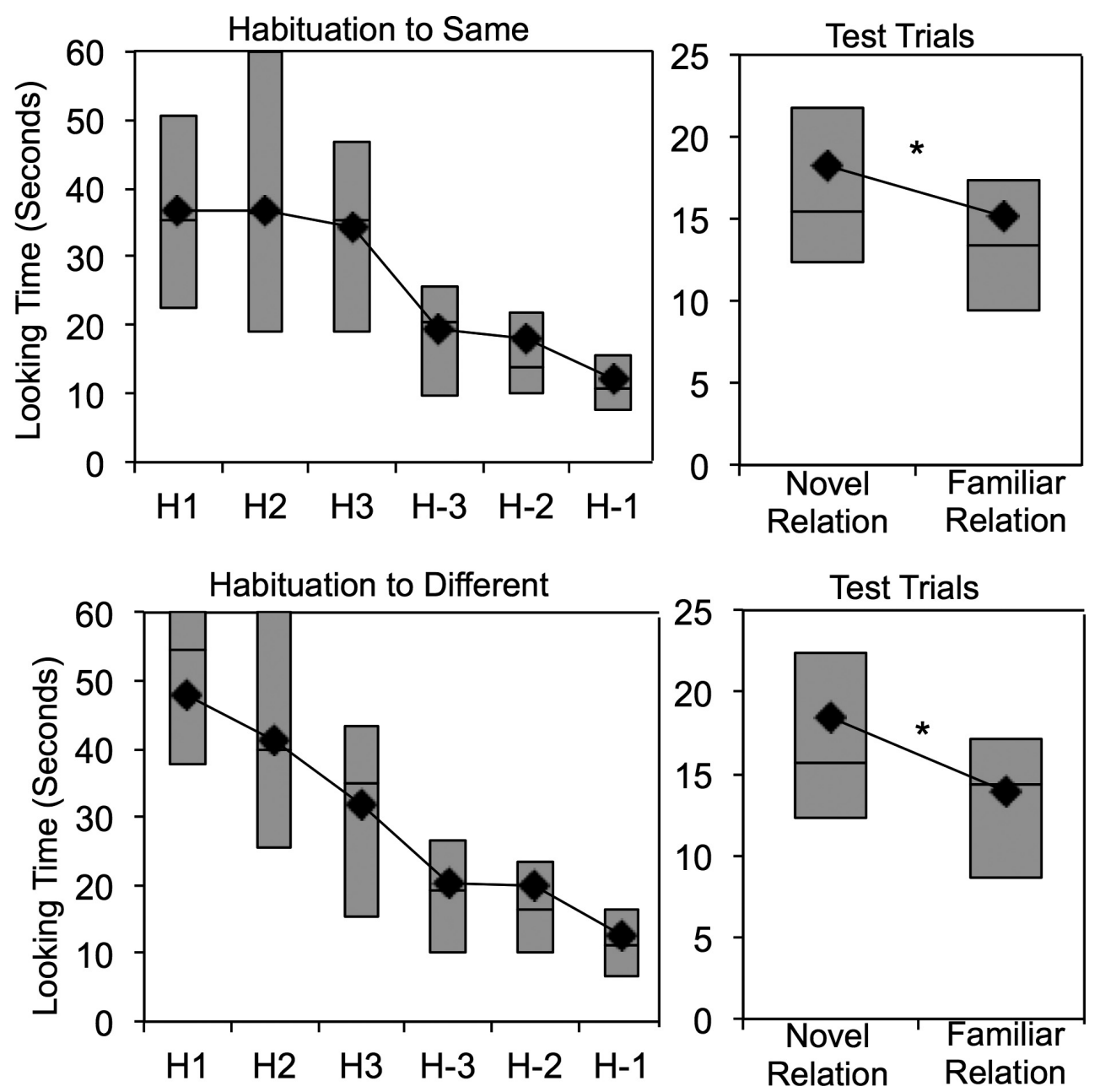

Figure 4. Box plots showing mean looking times during the habituation and test trials in Experiment 2. At test, preference for the novel relation was significant in both same and different conditions. The habituation graphs include results for the first three trials (H1, $\mathrm{H} 2$, H3) and the last three trials before the habituation criterion was met (H-3, H-2, H-1). Black diamonds represent means, the central line in each box is the median, and the upper and lower portions of each box represent the third and first quartiles respectively.

*Significant difference between novel and familiar test trials.

relation (novel, familiar), and prior experience (object experience, object experience + pair habituation, novel) revealed a significant effect of relation, $F(1,63)=20.01, p<.001, \eta^{2}=.24$, and a Relation $\times$ Prior Experience interaction, $F(2,62)=3.17$, $p=.049, \eta^{2}=.093$ (see Figure 5A). Further analyses revealed that infants looked significantly longer to the novel relation even with completely new objects (novel test trials), $F(1,63)=14.15, p<.001$, $\eta^{2}=.18$, indicating that they successfully generalized the abstract relation to new objects presented for the first time in test trials. In the trials in which attention was called to the individual objects (object experience test trials), the generalization of the relation was impeded; infants showed no significant difference in looking to the novel and familiar relations, $F(1,63)=0.114, p=.74, \eta^{2}=.002$. However, this adverse effect of object focus was mitigated by exposure to pairs containing these objects during habituation; on these trials, infants looked significantly longer to the novel relation (object experience + pair habituation test trials), $F(1,63)=8.70$, $p=.004, \eta^{2}=.12$.

To isolate the effect of prior experience and training we looked at the recovery scores of the first pair of tests (the first novel relation test trial and the first familiar relation test trial). Recovery scores are a standard looking-time analysis for habituation-dishabituation paradigms, involving three comparisons. The first is a $t$ test comparing the last habituation to the first novel test trial. If infants perceive the test event as novel, then there should be a significant increase in looking time from the last habituation trial to the novel test trial. The sec- 

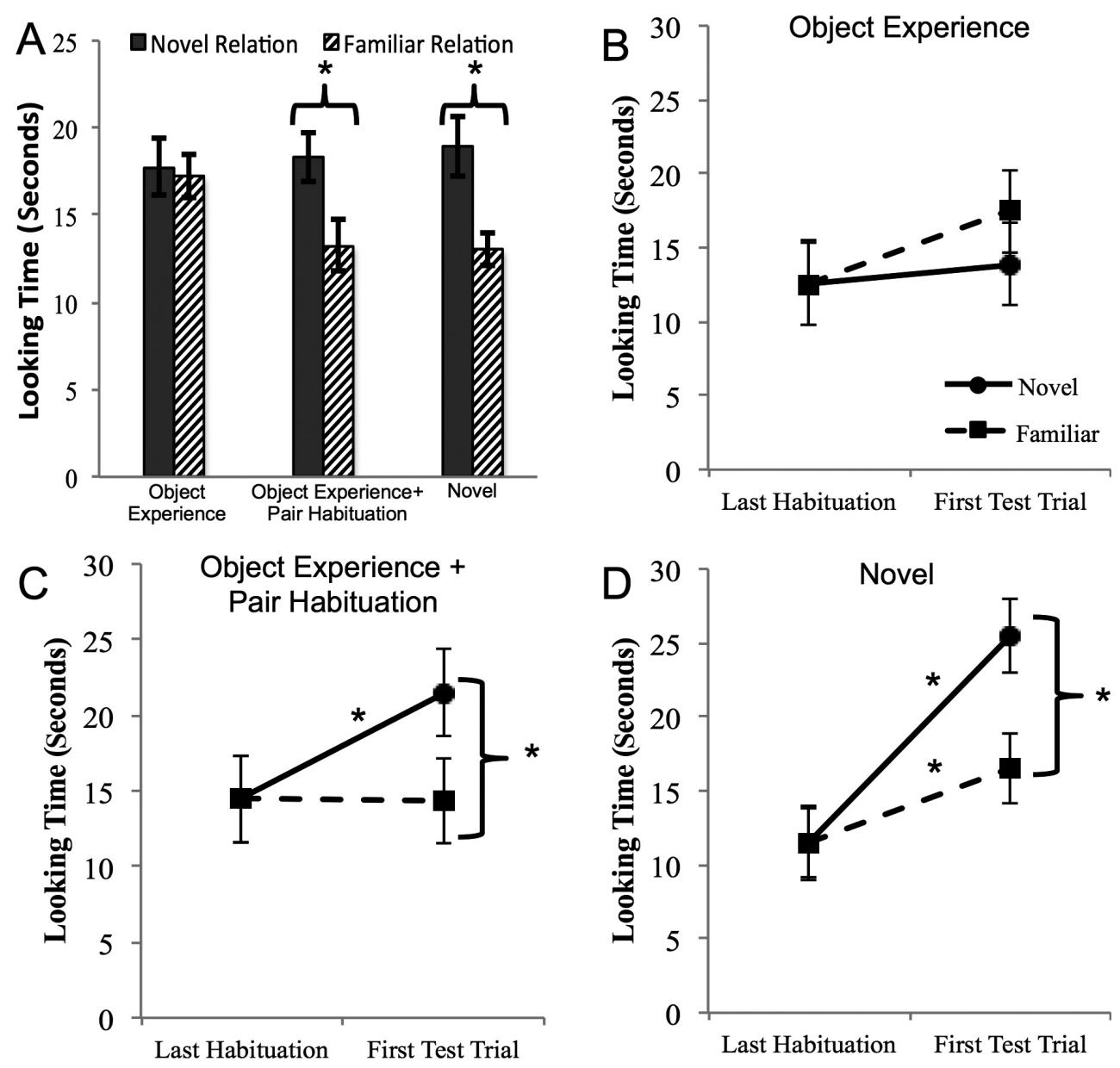

Figure 5. Looking time in Experiment 2. (A) The overall looking time to novel and familiar relations in the three types of test trials. The change in looking time from the last habituation to the first test pair (solid line for the novel event, dotted line for the familiar event) for (B) object experience, $(C)$ object experience + pair habituation, and (D) novel test trials.

$* p<.05$.

ond is a $t$ test comparing the last habituation to the first familiar test trial. If infants generalize their habituated response to the familiar test event, then looking time should not be statistically different between the last habituation trial and the familiar test trial. For completeness, the third analysis is a $t$ test comparing the novel and familiar test trials, which asks whether infants overall look significantly longer at the novel than at the familiar test trials. The recovery analysis goes beyond the significant interaction of relation by prior experience above, in that it focuses on the first test pair, removing the influence of prior test trials on the results. As we found no effect of condition (habituation to same or different) or age group (7- or 9month-olds) in the overall analysis of the prior experience effects, we collapse across these in the recovery scores analysis.
For infants who saw object experience as the first test trials, there was no change in looking time from the last habituation trial $(M=12.4 \mathrm{~s}$, $S D=9.5 \mathrm{~s})$ to the novel relation $(M=15.4 \mathrm{~s}$, $S D=11.7 \mathrm{~s}), t(22)=1.00, p=.33$, or from the last habituation trial to the familiar relation $(M=17.1 \mathrm{~s}$, $S D=10.8 s), t(22)=1.88, p=.08$. In addition, there was no overall difference between novel-relation and familiar-relation test trials, $t(22)=0.722$, $p=.478$ (see Figure 5B). This pattern suggests that when the first test trial consisted of high-salience objects, infants did not detect the same-different relation on that trial. In contrast, infants who saw object experience + pair habituation as their first test trials showed evidence of having encoded the relation. That is, they showed a significant increase from the last habituation trial $(M=14.71 \mathrm{~s}$, $S D=7.0 \mathrm{~s}$ ) to the novel-relation test trial 
$(M=22.2 \mathrm{~s}, S D=13.0 \mathrm{~s}), t(20)=2.45, p=.024$, but not to the familiar-relation test trial $(M=14.6 \mathrm{~s}$, $S D=13.3 \mathrm{~s}), \quad t(20)=0.49, p=.96$. Furthermore, there was a significant overall difference between the novel-relation and the familiar-relation test trials, $t(20)=2.16, p=.043$ (see Figure 5C). This is the classic dishabituation pattern. The infants dishabituated to the novel-relation event and generalized to the familiar-relation event. Infants who saw novel as their first test trials also showed significant recovery from the last habituation $(M=11.5 \mathrm{~s}$, $S D=7.4 \mathrm{~s})$ to the novel relation $(M=25.5 \mathrm{~s}$, $S D=17.9 \mathrm{~s}), t(19)=3.17, p=.005$. In addition, they showed significant recovery to the familiar relation $(M=16.5 \mathrm{~s}, \quad S D=7.5 \mathrm{~s}), \quad t(19)=2.48, \quad p=.023$. Importantly, there was a significant overall difference between the novel-relation and familiar-relation test trials, $t(19)=2.38, p=.028$. That infants dishabituated to both the novel-relation and familiar-relation test events suggests that they detected the objects as novel. However, the significant difference between the novel-relation and familiar-relation test events indicates that they also discriminated between the novel and familiar relations (see Figure 5D).

\section{Effects of Age}

To investigate whether there was an effect of age, we did an ANOVA with the within-subject variables of relation (novel vs. familiar) and test trial (first, second, or third pair) and the betweensubject factor of age (7 or 9 months). This revealed a marginal three-way interaction, $F(2,61)=2.78$, $p=.07, \eta^{2}=.08$. To make the analysis easier to perceive, we translated the relation factor into a difference score by subtracting the looking times at the familiar from the novel events for each test trial pair. These data are represented in Figure 6. They demonstrate that 9-month-old infants show a large difference in looking time (looking longer at the novel relation pair) on the first test pair, which diminishes over the course of test trials. In contrast, the 7-month-old infants show an opposite time course: They also look longer at the novel relation, but their difference in looking time increases across the test sets.

\section{Effects of Relation}

Our next question was whether infants would find it easier to align the same relation than the different relation. Perhaps surprisingly, we found no difference between these two conditions. As

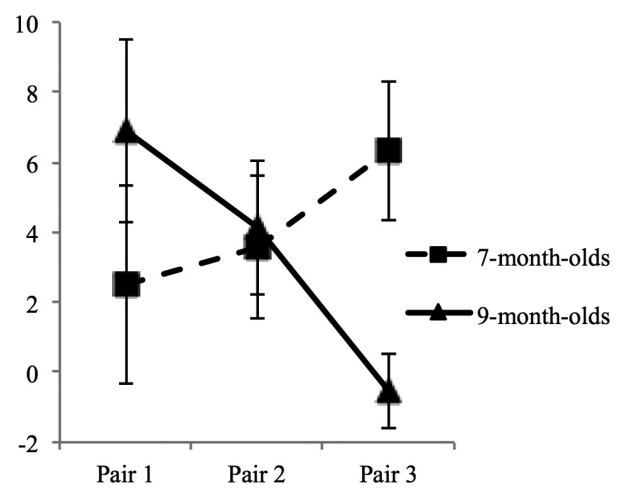

Figure 6. A line graph showing the difference score (looking time to the novel relation - looking time to the familiar relation) across the three pairs of test events and separated by age.

shown in Figure 3, infants detected the novel relation at test regardless of whether they were habituated to same or to different. Further analyses revealed that there was no difference in number of trials to meet the habituation criterion for same versus different $(M=7.42$ and 7.24 , respectively, range $=6-9), \quad F(1,62)<1, p=.57, \quad$ or in the amount of looking time that infants accumulated during habituation $(M=202 \mathrm{~s}, S D=75 \mathrm{~s}$; $M=218 \mathrm{~s}, S D=78 \mathrm{~s}$, respectively), $F(1,62)<1$, $p=.38$. Overall, we found no evidence that the same relation was easier to learn than the different relation.

This pattern of results was consistent and significant within each habituation condition. For the habituation to same condition, 23 of 31 infants $(74 \%)$ looked longer at the different test trials $(p=.011$, binomial comparison), and an ANOVA showed a significant main effect of test relation, $F(1,30)=5.01, p=.03, \eta^{2}=.14$. For the habituation to different condition, 26 of $33(79 \%)$ infants looked longer at the same test trials $(p=.001$, binomial comparison), and the ANOVA again showed a main effect of test relation, $F(1,32)=18.27$, $p<.001, \eta^{2}=.36$. We also found no reliable differences between same and different test trials when these relations were both novel, $F(2,61)<1$, or when they were both familiar, $F(2,61)<1$. Thus, we found no evidence in this study that same is easier to learn than different.

\section{Effects of Habituation Phase}

Thirty-eight percent of the infants met the habituation criterion in the minimum of six trials, $54 \%$ met the criterion in seven to nine trials, and $16 \%$ of infants did not meet the habituation criteria after the maximum of nine trials. On average, infants 
reached the habituation criterion after seven trials. Thus, infants typically saw three of the four training pairs a second time. An ANOVA of the accumulated looking during habituation reveal no main effect of age group, $F(1,60)<1$; no main effect of condition, $F(1,60)=1.13, p>.05$; and no interactions, $F(1,60)<1$. Infants accumulated an average of $210.6 \mathrm{~s}(S D=76.7 \mathrm{~s})$ of looking during the habituation phase. Additionally, there was no correlation between the duration of looking time during habituation trials and preference for the novel relation, $r(62)=-0.02$. A median split separating the shortest and the longest total looking times during habituation revealed that both groups looked significantly longer at the novel same-different relation than to the familiar relation (both Fs $>9.6$, both $p s<$.005). Thus, the amount of looking time during habituation had no impact on performance during test trials.

\section{Discussion}

The first question in this study was whether infants were capable of detecting and generalizing an abstract same-different relation. The results indicate that the answer is yes. Infants were able to generalize their learning to purely novel test pairs, showing that they had formed an abstract relation. Furthermore, infants habituated after just seven trials (on average) consisting of four distinct pairs plus repetitions, suggesting that the abstraction process was quite efficient. We infer that comparing across exemplars of the relation allowed the infant to abstract the common relational pattern-same or different. This pattern-comparing exemplars of a relation renders the relation more salient-is well established in studies with both adults (Gick \& Holyoak, 1983) and children (Childers \& Paik, 2009; Christie \& Gentner, 2010; Gentner et al., 2011; Kotovsky \& Gentner, 1996; Maguire et al., 2008). These findings suggest that this signature of analogical reasoning is present as early as 7 months of age.

We note that there is a way to save Possibility 1 here (that infants are born with abstract representations of same and different). It could be that the infants in Experiment 2 generalized better than those in Experiment 1 simply because they had more opportunities to encode the relation (and more time to encode overall) than did those in Experiment 1. Although we cannot rule out this possibility, the overall fit with predictions suggests that analogical generalization is the more parsimonious explanation.
The second question was whether there would be evidence of interference from attention to individual objects. Previous research on analogical learning has found that salient objects impede the structural alignment process (Gentner \& Rattermann, 1991; Gentner \& Toupin, 1986; Richland et al., 2006). To address this question, we systematically manipulated the infants' prior experience with the objects. Prior experience with the individual objects (object experience) interfered with the infants' ability to detect the same-different relation. However, this detrimental effect of prior object experience was overcome if infants then experienced those same objects within pairs during habituation. In that case, the infants successfully distinguished novel from familiar relations on the test pairs (object experience + pair habituation). Since infants also made this distinction for novel test pairs (consisting of objects they had not been seen before), their failure to show an effect for object experience test pairs is strong evidence that object focus interfered with their ability to detect the relation - consistent with findings demonstrating the detrimental effects of object focus on analogical processing.

Finally, we discovered an intriguing age effect. The older infants showed a large difference in looking time on the first test pair; then the difference attenuated over the subsequent pairs. The younger infants showed the opposite pattern. There was only a small difference in looking across the first two test trial types, but the difference increased across test trials. These differences suggest that (as one might have expected) the relational abstraction process had progressed further during habituation in the older infants than in the younger infants.

Our design also allowed us to ask whether performance would be better with the same relation than with the different relation. Intuitively, it seems possible that same is an elemental relation, while different may be represented as "not same" (Clark \& Chase, 1972; Hochmann, Mody, \& Carey, 2013), suggesting that children should more readily be able to abstract the same relation from experience than the different relation. However, prior evidence is mixed on this point (Addyman \& Mareschal, 2010; Smith, Redford, Haas, Coutinho, \& Couchman, 2008). In our study, we found no measurable difference in the likelihood of learning a same versus a different relation. An intriguing question is whether infants younger than 7 months would show an advantage for same over different. If so, then the idea that different is represented as not same might still be maintained. 
What was learned? After seeing a series of same or different exemplars in Experiment 2, infants represented an abstract relation of same or different that was not evident when they saw a single example in Experiment 1. How might this have happened? One possibility is that the infants had absolutely no notion of same and different relations before experiencing this series in Experiment 2. A more plausible explanation is that the infants were able to encode some initial form of the relation, at least for some of the pairs, but that these initial representations were highly context specific. In this case, the effect of structural alignment across examples was to lead the infants to rerepresent the relations in a more abstract way. On this account, infants would fail to apply the relation to a new pair after just one initial example (as we found in Experiment 1), but would be able to do so after aligning across examples (as we found in Experiment 2).

There is considerable evidence for this kind of sequential analogical abstraction (e.g., Gentner, Loewenstein \& Hung, 2007; Gentner et al., 2011; Haryu, Imai, \& Okada, 2011; Kotovsky \& Gentner, 1996). For example, in the Kotovsky and Gentner (1996) study, 4-year-old children were initially able to accurately match higher order spatial relational patterns (such as monotonic increase from left to right) within dimension but not across dimension. For example, they could match blue circles increasing in size with red squares increasing in size, but not with red squares increasing in darkness from left to right. But after experiencing several within-dimension trials (with no feedback), the children were able to perceive the cross-dimensional match. Gentner, Rattermann, Markman, and Kotovsky (1995) modeled these results by assuming that children initially represented the relations in a dimensionally specific manner (roughly "bigger than" and "darker than") and that structural alignment across examples prompted them to rerepresent the relations in a more abstract way (roughly "greater along a dimension than"). We think something like this could apply in the current study.

\section{General Discussion}

Analogical ability-the ability to perceive and reason about common relational patterns across disparate situations - is central to human intelligence. The available evidence indicates that humans are far better at representing and reasoning about relations than are other species. Recent theoretical accounts have argued that this difference in rela- tional ability is the central cognitive difference between humans and other primates (Gentner, 2003, 2010; Penn et al., 2008). The question of how human analogical ability develops is therefore important not only to theories of human development but also to accounts of comparative cognition and of how to characterize human intelligence.

In this article, we asked whether analogical ability is present in human infants, using the simplest and most basic relation - that of sameness or difference between two things. We proposed three broad possibilities: (a) that humans are born with analogical ability and a set of primitive relations for encoding early experiences, (b) that humans are born with analogical ability but not with a set of primitive relations, and (c) that analogical ability is not inherent in humans but emerges from linguistic and cultural experiences. We began by revisiting Tyrrell et al. (1991) influential study, which suggested that infants - even at 7 months of age - form an abstract same relation from just one exemplar of a same pair, and likewise for different. If indeed infants encode an abstract relation from a single exemplar, this would suggest that they already have a basic set of abstract relations, which can be applied without prior experience. These findings suggested a qualitative difference between humans and other primates, and have been widely cited in the primate literature. Because of the importance of this question, we first tried to replicate this finding.

Our data do not support this conclusion. In our attempted replication of the Tyrrell et al. (1991) study (Experiment 1), we found no evidence that prelinguistic human infants spontaneously encode abstract same-different matches from a single exemplar. The infants in our replication study showed a novelty response only when the familiar-relation pair was precisely identical (in objects as well as relation) to one that they had experienced before; there is no indication that they went beyond recognizing the identical item they had seen before. They failed to distinguish new same pairs from new different pairs, as would have been required to conclude that they already have represented an abstract same-different relation.

Our second question, given that 7- and 9-monthold infants do not immediately encode abstract same and different relations, was whether they could form these abstract relations via analogical learning processes. To address this, we asked whether the signatures of analogical learning seen in older children - the positive effects of multiple exemplars and the adverse effects of object focus-would be evident in infants' relational learning. We found that 7-month- 
old infants can indeed abstract a same or different relation across multiple exemplars. Although one exemplar was not sufficient (Experiment 1), the results of Experiment 2 showed that if infants are given a series of exemplars, they can form a same or different relation; that is, they can distinguish between same and different pairs even when these pairs are made up of totally novel objects. Only a modest number of exemplars was required for this learning. Infants saw four exemplars of same (or different) pairs, with two or three of them repeated, for a total of six to nine exemplars. Although we made no specific predictions here, this finding is consistent with the typical finding with older children, that comparing even a few exemplars of a relation can lead to significant learning.

Experiment 2 also bore out the second signature of analogical learning-namely, that object focus interferes with relational matching. When infants saw pairs consisting of objects that had been demonstrated as single objects in the playroom prior to the study, they failed to show a distinction between same and different pairs unless they had subsequently seen the objects in pairs during habituation. This is consistent with other findings demonstrating that a focus on individual objects can disrupt relational processing (Casasola, 2005; Christie \& Gentner, 2010; Gentner \& Toupin, 1986; Maguire et al., 2008; Richland et al., 2006). It appears that there is continuity in the signatures of relational learning in the earliest evidence of this ability.

Our findings support the broad position that humans are born with analogical skills and use these skills to extract relational information from experiences. Thus, they argue against the third possibility suggested earlier-that analogical ability emerges developmentally as an amalgam of other cognitive processes, rather than being an inherent cognitive capacity. Of course, this is not to suggest that this ability is fully developed at an early age. Clearly, there are developmental changes and influences from language and cultural experiences. Nevertheless these are the first data that demonstrate that signatures of the analogical processing are evident early in development.

\section{Broader Implications and Future Directions}

Returning to the question of how to characterize human relational ability, we found no support for the strong view that humans are endowed with a set of abstract relational representations. Rather, our findings suggest that humans are born with considerable analogical processing ability, with which they can detect abstract relations. This characterization is consistent with there being a continuum of analogical ability between our species and other apes, rather than a sharp dichotomy. As we see it, the evidence from primate studies suggests that other apes possess some analogical reasoning ability, though to a substantially lesser degree than humans (Christie, Gentner, Call, \& Haun, 2015; Haun \& Call, 2009; Premack, 1983; Thompson et al., 1997). Indeed, recent studies have suggested that even more distant relatives (hooded crows) have some analogical ability and can pass the relational-match-to-sample test under some circumstances (Smirnova, Zorina, Obozova, \& Wasserman, 2015).

Our findings also bear on the role of language in analogical learning. Gentner and colleagues have suggested that acquiring and using relational language is instrumental in the development of analogical processing (Christie \& Gentner, 2014; Gentner, 2010; Gentner \& Rattermann, 1991; Gentner et al., 2011). Our results clearly place limits on these claims. The infants in our study were able to carry out analogical generalization of a same-different relation prior to learning terms for same and different. We can infer that analogical ability does not depend on the possession of relational language. However, research with older children shows that language can augment our relational capacity, by inviting comparison (Namy \& Gentner, 2002) and by heightening attention to particular relations (Casasola, 2005; Gentner, Özyürek, Gürcanli, \& GoldinMeadow, 2013). Thus, it is an open question whether we might see language effects later in development. We know that relational terms are acquired later than object-reference terms (Bornstein et al., 2004; Gentner, 1982; Gentner \& Boroditsky, 2009); indeed, infants do not appear to understand relational words until the end of the 1st year (Bergelson \& Swingley, 2013). Thus, future research should investigate whether and when we might see language effects for same-different processing in infants.

Earlier we raised the question of whether infants' abilities in artificial grammar tasks could be related to the analogical learning processes studied here. We think it quite possible that some of the same processes are involved. Artificial grammar tasks capture two crucial requirements for grammar learning: first, the ability to learn sequential transition probabilities (Saffran et al., 1996), and second, the ability to extract and learn parallel structure across a set of exemplars (Marcus, 2000; see also Gomez \& Gerken, 2000). It is this second ability, often termed rule learning, that is most closely con- 
nected with analogical generalization. That infants can learn this kind of general structure was demonstrated by Marcus et al. (1999) in their seminal study showing that 7-month-olds exposed to a series of exemplars such as pa ti pa and li na li formed an abstract ABA structure - as evidenced by their showing a novelty response to a new pattern (e.g., wo wo fe) but not to an old pattern (wo fe wo).

Is this kind of relational generalization a product of analogical learning? There is some suggestive evidence for this speculation. First, the same kind of generalization patterns have been found for visual stimuli (Johnson et al., 2009; Saffran et al., 2007). This is consistent with the operation of a domain-general learning process such as analogy, rather than a dedicated language-specific process. Second, studies by Gerken (2006) show that when the exemplars all contain the same extra element, the infants' generalization retains that elementconsistent with analogical learning patterns, which typically show conservative generalization, especially early in learning. A third line of evidence, also noted above, is that we have been able to simulate the Marcus et al. (1999) study with a computational simulation of analogical generalization (Kuehne et al., 2000). Unlike most attempts to simulate these results, the analogical generalization mechanism requires only the same 48 exemplars given to the infants, and can succeed even with noise added to the data. Of course, these are only plausibility arguments. An important future direction will be to test directly whether analogical generalization processes operate in artificial grammar learning.

Another open question is how to square our claims concerning the continuity of relational learning processes with the fact that even $2 \frac{1}{2}$-year-old children have difficulty with same-different relational matching (Christie \& Gentner, 2014). One possible explanation lies in the difference between the tasks typically given to older and younger children. For example, there is evidence that 18-monthold children can succeed in abstracting same-different relations across examples in service of a causal learning task (Walker \& Gopnik, 2014).

Our findings also raise questions for future infant research. First, what is the earlier course of relational learning? Would we see analogical learning even among much younger infants, and if so, would they require more extensive training (e.g., more exemplars, more repetitions or longer exposure times) to abstract relations? Research with newborn infants by Gervain, Berent, and Werker (2012) suggests that some sensitivity to relational patterns may appear very early. Using the functional near-infrared spectroscopy technique, they found that newborns formed a different neural response to $A A B$ patterns than to $A B B$ patterns after exposure to blocks of syllables in the two patterns. Whether and when this ability would allow infants to generalize to distinguish new instances of one pattern from another is an intriguing question.

In conclusion, our findings show that analogical ability is present in infants by at least 7 months of age. Our results also suggest that there is continuity in the analogical learning process across development, and leave open the possibility of continuity across species.

\section{References}

Addyman, C., \& Mareschal, D. (2010). The perceptual origins of the abstract same/different concept in human infants. Animal Cognition, 13, 817-833. doi:10.1007/ s10071-010-0330-0

Aslin, R. N., \& Newport, E. L. (2012). Statistical learning: From acquiring specific items to forming general rules. Current Directions in Psychological Science, 21, 170-176. doi:10.1177/0963721412436806

Baillargeon, R., Li, J., Gertner, Y., \& Wu, D. (2011). How do infants reason about physical events? In U. Goswami (Ed.), The Wiley-Blackwell handbook of childhood cognitive development (2nd ed., pp. 11-48). Oxford, UK: Blackwell. doi:10.1002/9781444325485.ch1

Bergelson, E., \& Swingley, D. (2013). The acquisition of abstract words by young infants. Cognition, 127, 391397. doi:10.1016/j.cognition.2013.02.011

Bornstein, M. H., Cote, L. R., Maital, S., Painter, K., Park, S.-Y., Pascual, L., . . Vyt, A. (2004). Crosslinguistic analysis of vocabulary in young children: Spanish, Dutch, French, Hebrew, Italian, Korean, and American English. Child Development, 75, 1115-1139. doi:10.1111/ j.1467-8624.2004.00729.x

Casasola, M. (2005). When less is more: How infants learn to form an abstract categorical representation of support. Child Development, 76, 279-290. doi:10.1111/j.14678624.2005.00844.x

Casasola, M., \& Cohen, L. B. (2002). Infant categorization of containment, support, and tight-fit spatial relationships. Developmental Science, 5, 247-264. doi:10.1111/ 1467-7687.00226

Childers, J. B., \& Paik, J. H. (2009). Korean- and Englishspeaking children use cross-situational information to learn novel predicate terms. Journal of Child Language, 36, 201-224. doi:10.1017/s0305000908008891

Christie, S., \& Gentner, D. (2010). Where hypotheses come from: Learning new relations by structural alignment. Journal of Cognition and Development, 11, 356-373 doi:10.1080/15248371003700015 
Christie, S., \& Gentner, D. (2014). Language helps children succeed on a classic analogy task. Cognitive Science, 38, 383-397. doi:10.1111/cogs.12099

Christie, S., Gentner, D., Call, J., \& Haun, D. (2015). Sensitivity to relational similarity and object similarity in apes and children.

Clark, E. V. (1973). What's in a word? On the child's acquisition of semantics in his first language. In T. E. Moore (Ed.), Cognitive development and the acquisition of language (pp. 65-110). New York, NY: Academic Press.

Clark, H. H., \& Chase, W. G. (1972). On the process of comparing sentences against pictures. Cognitive Psychology, 3, 472-517. doi:10.1016/0010-0285(72)90019-9

DeLoache, J. S. (1995). Early symbol understanding and use. In D. L. Medin (Ed.), The psychology of learning and motivation: Advances in research and theory (Vol. 33, pp. 65-114). San Diego, CA: Academic Press.

Dewar, K. M., \& Xu, F. (2010). Induction, overhypothesis, and the origin of abstract knowledge: Evidence from 9month-old infants. Psychological Science, 21, 1871-1877. doi:10.1093/acprof:oso/9780199216895.003.0011

Doumas, L. A. A., Hummel, J. E., \& Sandhofer, C. M. (2008). A theory of the discovery and predication of relational concepts. Psychological Review, 115, 1-43. doi:10.1037/0033-295x.115.1.1

ELAN. (2007). ELAN. Nijmegen, Netherlands: Language Archiving Technologies.

Elman, J. (1998). Generalization, simple recurrent networks, and the emergence of structure. Presented at the annual conference of the Cognitive Science Society, Madison, WI.

Fagot, J., \& Thompson, R. K. R. (2011). Generalized relational matching by guinea baboons (Papio papio) in two-by-two-item analogy problems. Psychological Science, 22, 1304-1309. doi:10.1177/0956797611422916

Ferguson, R. W. (1994). MAGI: Analogy-based encoding using regularity and symmetry. In A. Ram \& K. Eiselt (Eds.), Proceedings of the Sixteenth Annual Conference of the Cognitive Science Society (pp. 283-288). New York, NY: Psychology Press.

Flemming, T. M., Beran, M. J., \& Washburn, D. A. (2007). Disconnect in concept learning by rhesus monkeys (Macaca mulatta): Judgment of relations and relationsbetween-relations. Journal of Experimental Psychology: Animal Behavior Processes, 33, 55-63. doi:10.1037/00977403.33.1.55

Gentner, D. (1982). Why nouns are learned before verbs: Linguistic relativity versus natural partitioning. In S. A. Kuczaj (Ed.), Language development: Language thought and culture (Vol. 2, pp. 301-334). Hillsdale, NJ: Erlbaum.

Gentner, D. (1988). Metaphor as structure mapping: The relational shift. Child Development, 59, 47-59. doi:10.2307/11303

Gentner, D. (2003). Why we're so smart. In D. Gentner \& S. Goldin-Meadow (Eds.), Language in mind: Advances in the study of language and thought (pp. 195-235). Cambridge, MA: MIT Press.
Gentner, D. (2010). Bootstrapping the mind: Analogical processes and symbol systems. Cognitive Science, 34, 752-775. doi:10.1111/j.1551-6709.2010.01114.x

Gentner, D., Anggoro, F. K., \& Klibanoff, R. S. (2011). Structure mapping and relational language support children's learning of relational categories. Child Development, 82, 1173-1188. doi:10.1111/j.1467-8624.2011. 01599.x

Gentner, D., \& Boroditsky, L. (2009). Early acquisition of nouns and verbs: Evidence from Navajo. In V. Gathercole (Ed.), Routes to language: Studies in honor of Melissa Bowerman (pp. 5-36). New York, NY: Psychology Press.

Gentner, D., Holyoak, K. J., \& Kokinov, B. N. (2001). The analogical mind: Perspectives from cognitive science. Cambridge, MA: MIT Press.

Gentner, D., Loewenstein, J., \& Hung, B. (2007). Comparison facilitates children's learning of names for parts. Journal of Cognition and Development, 8, 285-307.

Gentner, D., \& Medina, J. (1998). Similarity and the development of rules. Cognition, 65, 263-297. doi:10.1016/ s0010-0277(98)00002-x

Gentner, D., \& Namy, L. (1999). Comparison in the development of categories. Cognitive Development, 14, 487513. doi:10.1016/s0885-2014(99)00016-7

Gentner, D., Özyürek, A., Gürcanli, Ö., \& Goldin-Meadow, S. (2013). Spatial language facilitates spatial cognition: Evidence from children who lack language input. Cognition, 127, 318-330. doi:10.1016/j.cognition.2013.01.003

Gentner, D., \& Rattermann, M. J. (1991). Language and the career of similarity. In S. A. Gelman \& J. P. Byrnes (Eds.), Perspectives on language and thought: Interrelations in development (pp. 225-277). New York, NY: Cambridge University Press.

Gentner, D., Rattermann, M. J., Markman, A. B., \& Kotovsky, L. (1995). Two forces in the development of relational similarity. In T. J. Simon \& G. S. Halford (Eds.), Developing cognitive competence: New approaches to process modeling (pp. 263-313). Hillsdale, NJ: Erlbaum.

Gentner, D., \& Toupin, C. (1986). Systematicity and surface similarity in the development of analogy. Cognitive Science, 10, 277-300. doi:10.1207/s15516709cog1003_2

Gerken, L. (2006). Decisions, decisions: Infant language learning when multiple generalizations are possible. Cognition, 98, B67-B74. doi:10.1016/j.cognition.2005.03.003

Gervain, J., Berent, I., \& Werker, J. F. (2012). Binding at birth: The newborn brain detects identity relations and sequential position in speech. Journal of Cognitive Neuroscience, 24, 564-574. doi:10.1162/jocn_a_00157

Gick, M. L., \& Holyoak, K. J. (1983). Schema induction and analogical transfer. Cognitive Psychology, 15, 1-38. doi:10.1016/0010-0285(83)90002-6

Giurfa, M., Zhang, S., Jennett, A., Menzel, R., \& Srinivasan, M. V. (2001). The concepts of "sameness" and "difference" in an insect. Nature, 410, 930-933. doi:10.1038/ 35073582

Gomez, R. L., \& Gerken, L. A. (1999). Artificial grammar learning by 1-year-olds leads to specific and abstract knowledge. Cognition, 70, 109-135. 
Gomez, R. L., \& Gerken, L. (2000). Infant artificial language learning and language acquisition. Trends in Cognitive Sciences, 4, 178-186. doi:10.1016/s1364-6613(00) 01467-4

Halford, G. S. (1992). Analogical reasoning and conceptual complexity in cognitive development. Human Development, 35, 193-217. doi:10.1159/000277167

Haryu, E., Imai, M., \& Okada, H. (2011). Object similarity bootstraps young children to action-based verb extensions. Child Development, 82, 674-686. doi:10.1111/ j.1467-8624.2010.01567.x

Haun, D. B. M., \& Call, J. (2009). Great apes' capacities to recognize relational similarity. Cognition, 110, 147-159. doi:10.1016/j.cognition.2008.10.012

Hespos, S. J., \& Baillargeon, R. (2001). Infants' knowledge about occlusion and containment events: A surprising discrepancy. Psychological Science, 12, 141-147. doi:10.1111/1467-9280.00324

Hespos, S. J., \& Baillargeon, R. (2006). Decalage in infants' knowledge about occlusion and containment events: Converging evidence from action tasks. Cognition, 99, B31-B41. doi:10.1016/j.cognition.2005.01.010

Hespos, S. J., Grossman, S. R., \& Saylor, M. M. (2010). Infants' ability to parse continuous actions: Further evidence. Neural Networks, 23, 1026-1032. doi:10.1016/ j.neunet.2010.07.010

Hespos, S. J., \& Piccin, T. B. (2009). To generalize or not to generalize: Spatial categories are influenced by physical attributes and language. Developmental Science, 12, 88-95. doi:10.1111/j.1467-7687.2008.00749.x

Hespos, S. J., \& Spelke, E. S. (2004). Conceptual precursors to language. Nature, 430, 453-456. doi:10.1038/nature02634

Hochmann, J., Mody, S., \& Carey, S. (2013). Same and different relations in match- and mismatch-to-sample. Presented at the Cognitive Development Society, Memphis, TN.

James, W. (1890). The principles of psychology. Cambridge, MA: Harvard University Press.

Johnson, S. P., Fernandes, K. J., Frank, M. C., Kirkham, N., Marcus, G., Rabagliati, H., \& Slemmer, J. A. (2009). Abstract rule learning for visual sequences in 8- and 11-month-olds. Infancy, 14, 2-18. doi:10.1080/ 15250000802569611

Kirkham, N. Z., Slemmer, J. A., \& Johnson, S. P. (2002). Visual statistical learning in infancy: Evidence for a domain general learning mechanism. Cognition, 83, B35-B42. doi:10.1016/s0010-0277(02)00004-5

Kotovsky, L., \& Gentner, D. (1996). Comparison and categorization in the development of relational similarity. Child Development, 67, 2797-2822. doi:10.2307/1131753

Kuehne, S. E., Gentner, D., \& Forbus, K. D. (2000). Modeling infant learning via symbolic structural alignment. In L. R. Gleitman \& A. K. Joshi (Eds.), Proceedings of the Twenty-Second Annual Conference of the Cognitive Science Society (pp. 286-291). Mahwah, NJ: Erlbaum.

Maguire, M. J., Hirsh-Pasek, K., Golinkoff, R. M., \& Brandone, A. C. (2008). Focusing on the relation: Fewer exemplars facilitate children's initial verb learning and extension. Developmental Science, 11, 628-634. doi:10.1 111/j.1467-7687.2008.00707.x

Marcus, G. F. (2000). Pabiku and Ga Ti Ga: Two mechanisms infants use to learn about the world. Current Directions in Psychological Science, 9, 145-147. doi:10.1111/1467-8721.00080

Marcus, G. F., Fernandes, K. J., \& Johnson, S. P. (2007). Infant rule learning facilitated by speech. Psychological Science, 18, 387-391. doi:10.1111/j.1467-9280.2007. 01910.x

Marcus, G. F., Vijayan, S., Rao, S. B., \& Vishton, P. M. (1999). Rule learning by seven-month-old infants. Science, 283, 77-80. doi:10.1126/science.283.5398.77

Markman, A. B., \& Gentner, D. (1993). Structural alignment during similarity comparisons. Cognitive Psychology, 25, 431-467. doi:10.1006/cogp.1993.1011

McDonough, L., Choi, S., \& Mandler, J. M. (2003). Understanding spatial relations: Flexible infants, lexical adults. Cognitive Psychology, 46, 229-259. doi:10.1016/ s0010-0285(02)00514-5

Mix, K. S. (2008). Children's equivalence judgments: Crossmapping effects. Cognitive Development, 23, 191203. doi:10.1016/j.cogdev.2007.03.001

Namy, L. L., \& Gentner, D. (2002). Making a silk purse out of two sow's ears: Young children's use of comparison in category learning. Journal of Experimental Psychology: General, 131, 5-15. doi:10.1037/0096-3445.131.1.5

Paik, J. H., \& Mix, K. S. (2006). Preschoolers' use of surface similarity in object comparisons: Taking context into account. Journal of Experimental Child Psychology, 95, 194-214. doi:10.1016/j.jecp.2006.06.002

Palmer, S. E. (1977). Hierarchical structure in perceptual representation. Cognitive Psychology, 9, 441-474. doi:10.1037/e666602011-014

Penn, D. C., Holyoak, K. J., \& Povinelli, D. J. (2008). Darwin's mistake: Explaining the discontinuity between human and nonhuman minds. Behavioral and Brain Sciences, 31, 109-130. doi:10.1017/s0140525x 08003798

Pinto, J. P. (1996). Xhab: Experimental control software for MS-DOS (Version 6.5) [Computer software]. Palo Alto, CA: Author.

Premack, D. (1983). The codes of man and beasts. Behavioral and Brain Sciences, 6, 125-167. doi:10.1017/ s0140525x00015077

Quinn, P. C., Cummins, M., Kase, J., Martin, E., \& Weissman, S. (1996). Development of categorical representations for above and below spatial relations in $3-$ to 7-month-old infants. Developmental Psychology, 32, 942950. doi:10.1037/0012-1649.32.5.942

Richland, L. E., Morrison, R. G., \& Holyoak, K. J. (2006). Children's development of analogical reasoning: Insights from scene analogy problems. Journal of Experimental Child Psychology, 94, 249-271. doi:10.1016/ j.jecp.2006.02.002

Robinson, J. S. (1955). The sameness-difference discrimination problem in chimpanzee. Journal of Comparative 
and Physiological Psychology, 48, 195. doi:10.1037/ h0042463

Saffran, J. R., Aslin, R. N., \& Newport, E. L. (1996). Statistical learning by 8-month-old infants. Science, 274, 1926-1928. doi:10.1126/science.274.5294.1926

Saffran, J. R., Pollak, S. D., Seibel, R. L., \& Shkolnik, A. (2007). Dog is a dog is a dog: Infant rule learning is not specific to language. Cognition, 105, 669-680. doi:10.1016/j.cognition.2006.11.004

Sinha, P., Balas, B., Ostrovsky, Y., \& Russell, R. (2006). Face recognition by humans: Nineteen results all computer vision researchers should know about. Proceedings of the Institute of Electrical and Electronics Engineers, 94, 1948-1962. doi:10.1109/jproc.2006.884093

Smirnova, A., Zorina, Z., Obozova, T., \& Wasserman, E. (2015). Crows spontaneously exhibit analogical reasoning. Current Biology, 25, 256-260.

Smith, J. D., Redford, J. S., Haas, S. M., Coutinho, M. V., \& Couchman, J. J. (2008). The comparative psychology of same-different judgments by humans (Homo sapiens) and monkeys (Macaca mulatta). Journal of Experimental Psychology: Animal Behavior Processes, 34, 361-374. doi:10.1037/0097-7403.34.3.361

Smith, L. B. (1984). Young children's understanding of attributes and dimensions: A comparison of conceptual and linguistic measures. Child Development, 55, 363-380. doi:10.2307/1129949

Thibaut, J.-P., French, R., \& Vezneva, M. (2010). The development of analogy making in children: Cognitive load and executive functions. Journal of Experimental Child Psychology, 106, 1-19. doi:10.1016/j.jecp.2010. 01.001

Thompson, R. K., \& Oden, D. L. (1995). A profound disparity revisited: Perception and judgment of abstract identity relations by chimpanzees, human infants, and monkeys. Behavioral Processes, 35, 149-161. doi:10.1016/ 0376-6357(95)00048-8

Thompson, R. K. R., \& Oden, D. L. (2000). Categorical perception and conceptual judgments by nonhuman primates: The paleological monkey and the analogical ape. Cognitive Science, 24, 363-396. doi:10.1207/ s15516709cog2403_2

Thompson, R. K. R., Oden, D. L., \& Boysen, S. T. (1997). Language-naive chimpanzees (Pan troglodytes) judge relations between relations in a conceptual matchingto-sample task. Journal of Experimental Psychology: Animal Behavior Processes, 23, 31-43. doi:10.1037/00977403.23.1.31

Tyrrell, D. J., Stauffer, L. B., \& Snowman, L. G. (1991). Perception of abstract identity/difference relationships by infants. Infant Behavior and Development, 14, 125-129. doi:10.1016/0163-6383(91)90059-2

Tyrrell, D. J., Zingardo, M. C., \& Minard, K. L. (1993). Learning and transfer of identity difference relationships by infants. Infant Behavior and Development, 16, 43-52. doi:10.1016/0163-6383(93)80027-6

Walker, C. M., \& Gopnik, A. (2014). Toddlers infer higher-order relational principles in causal learning. Psychological Science, 25, 161-169. doi:10.1177/ 0956797613502983

Wang, S.-H., \& Baillargeon, R. (2008). Can infants be "taught" to attend to a new physical variable in an event category? The case of height in covering events. Cognitive Psychology, 56, 284-326. doi:10.1016/j.cogpsych.2007.06.003

Wasserman, E. A., \& Young, M. E. (2010). Same-different discrimination: The keel and backbone of thought and reasoning. Journal of Experimental Psychology: Animal Behavior Processes, 36, 3-22. doi:10.1037/a0016327

Wright, A. A., \& Katz, J. S. (2006). Mechanisms of same/ different concept learning in primates and avians. Behavioural Processes, 72, 234-254. doi:10.1016/j.beproc.2006.03.009

Zentall, T. R., Wasserman, E. A., Lazareva, O. F., Thompson, R. K. R., \& Rattermann, M. J. (2008). Concept learning in animals. Comparative Cognition $\mathcal{E}$ Behavior Review, 3, 13-45. doi:10.3819/ccbr.2008.30002 